\title{
Wide-ridge metal-metal terahertz quantum cascade lasers with high-order lateral mode suppression
}

\section{Citation}

Fan, Jonathan A., Mikhail A. Belkin, Federico Capasso, Suraj P. Khanna, Mohamed Lachab, A. Giles Davies, and Edmund H. Linfield. 2008. “Wide-Ridge Metal-Metal Terahertz Quantum Cascade Lasers with High-Order Lateral Mode Suppression." Applied Physics Letters 92 (3): 31106. https://doi.org/10.1063/1.2835202.

\section{Permanent link}

http://nrs.harvard.edu/urn-3:HUL.InstRepos:41372468

\section{Terms of Use}

This article was downloaded from Harvard University's DASH repository, and is made available under the terms and conditions applicable to Other Posted Material, as set forth at http:// nrs.harvard.edu/urn-3:HUL.InstRepos:dash.current.terms-of-use\#LAA

\section{Share Your Story}

The Harvard community has made this article openly available.

Please share how this access benefits you. Submit a story.

\section{Accessibility}




\title{
Wide-ridge metal-metal terahertz quantum cascade lasers with high-order lateral mode suppression
}

\author{
Jonathan A. Fan, ${ }^{\text {a) }}$ Mikhail A. Belkin, and Federico Capasso ${ }^{\text {b) }}$ \\ Harvard School of Engineering and Applied Sciences, Harvard University, Cambridge, \\ Massachusetts 02138, USA \\ Suraj P. Khanna, Mohamed Lachab, A. Giles Davies, and Edmund H. Linfield \\ School of Electronic and Electrical Engineering, University of Leeds, Leeds LS2 9JT, United Kingdom
}

(Received 20 November 2007; accepted 24 December 2007; published online 23 January 2008)

\begin{abstract}
Terahertz quantum cascade lasers with wide-ridge metal-metal waveguides are prone to lasing in high-order lateral modes, which reduce the maximum light output power from these devices. We have demonstrated, theoretically and experimentally, that the output power can be improved severalfold by introducing "side absorbers" into the waveguide structure, which enforce lasing in the $\mathrm{TM}_{00}$ mode with minor temperature performance deterioration. Lasers without side absorbers operate up to $168 \mathrm{~K}$, a current record for devices processed using indium/gold wafer bonding.

(C) 2008 American Institute of Physics. [DOI: 10.1063/1.2835202]
\end{abstract}

Terahertz quantum cascade lasers (QCLs) are an emergent compact source of narrowband terahertz radiation in the wavelength range of $60-350 \mu \mathrm{m}(5-0.85 \mathrm{THz}) .{ }^{1}$ In order to achieve lasing, the laser mode requires strong confinement within the active region. This challenge is addressed by the use of surface plasmon waveguides, of which two types have been reported: the semi-insulating surface-plasmon (SI-SP) waveguide and the metal-metal (MM) waveguide. The SI-SP waveguide $^{2}$ utilizes a metal film on top of the QCL active region and provides mode confinement of $\sim 15 \%-50 \%{ }^{3}$ The MM waveguide ${ }^{4,5}$ comprises metal films on both sides of the active region and provides mode confinement of nearly $100 \% .^{3-5}$

The MM waveguide design, compared to the SI-SP waveguide design, empirically provides the best temperature performance for terahertz QCLs, ${ }^{5,6}$ but it suffers from poor radiation out-coupling efficiency and a highly divergent farfield profile because the mode is confined to subwavelength dimensions. ${ }^{3}$ While some applications, such as heterodyne detection, do not require high output power, other applications such as imaging ${ }^{7,8}$ require both a good far-field profile and high output power. These parameters can be improved by using wide-ridge $(>100 \mu \mathrm{m})$ waveguides; however, we will show that wide-ridge MM waveguides are prone to high-order lateral mode operation. This is undesirable because it yields a highly divergent, multiple-lobe far-field profile. In addition, as we will further analyze, the facet reflectivity for higher order lateral modes is significantly higher than that for the $\mathrm{TM}_{00}$ mode, yielding reduced radiation outcoupling efficiency.

A number of approaches addressing the problem of poor terahertz outcoupling in MM terahertz QCLs have been proposed, including the use of a silicon microlens at the waveguide facet $^{9}$ and surface-emission schemes that utilize second-order diffraction gratings ${ }^{10,11}$ or two-dimensional photonic crystal structures. ${ }^{12}$ However, the positioning of a microlens onto the waveguide facet is difficult, ${ }^{9}$ and edge-

\footnotetext{
${ }^{a)}$ Electronic mail: jfan@fas.harvard.edu.

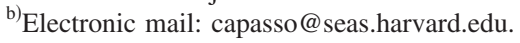

emitting lasers are significantly easier to fabricate than surface-emitting lasers.

In this letter, we demonstrate lasing mode control in wide-ridge MM terahertz QCLs. We fabricate 150- $\mu \mathrm{m}$-wide edge emitting MM terahertz QCLs operating at $3 \mathrm{THz}$ with thin strips of highly doped GaAs exposed along the ridge waveguide edges [the "side absorbers," Fig. 1(a)]. These devices operate in the $\mathrm{TM}_{00}$ mode and demonstrate a threefold increase in power output with little temperature degradation compared to devices without side absorbers. We note that this laser mode control technique may be used to improve the outcoupling efficiency and the far-field profile for edgeemitting devices equipped with microlenses, and can be implemented in surface-emitting MM terahertz QCLs with
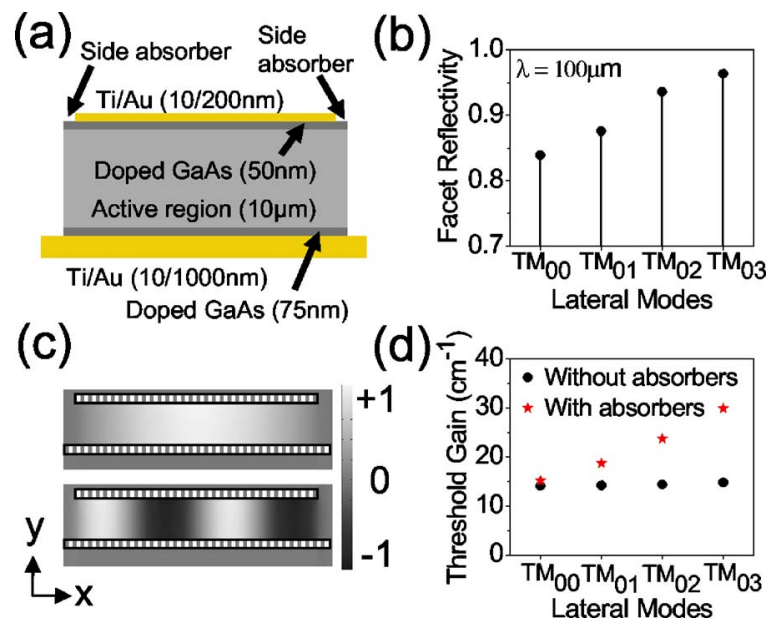

FIG. 1. (Color online) (a) Cross-sectional schematic diagram of the MM waveguide QCL with side absorbers. Layer thicknesses are marked in parentheses; the GaAs layers are doped to $5 \times 10^{18} \mathrm{~cm}^{-3}$. The side absorbers consist of exposed plasmon layer at the edges of the top metal cladding and are marked with arrows. (b) Facet reflectivity as a function of lateral mode order for a $10-\mu \mathrm{m}$-thick and $150-\mu \mathrm{m}$-wide MM waveguide. (c) Crosssection plots of the $\mathrm{H}_{x}$ component of two lateral waveguide modes. The upper plot is the $\mathrm{TM}_{00}$ mode and the lower plot is the $\mathrm{TM}_{03}$ mode. The metal waveguide claddings are shaded in vertical stripes. (d) Threshold gain as a function of lateral mode order for laser structures with and without side absorbers. 
wide ridges, which suffer from high-order lateral mode lasing. ${ }^{10,11}$ We also report devices without side absorbers that operate up to a maximum temperature of $168 \mathrm{~K}$, which constitutes the highest operating temperature achieved to date with MM terahertz QCLs processed using In/Au wafer bonding.

To understand the modal structure of the emission in wide-ridge MM devices, we calculated the lasing threshold for various lateral modes using COMSOL MULTIPHYSICS. First, we calculated the facet reflectivity for different lateral modes using three-dimensional simulations. We assumed a lossless active region with a refractive index of 3.5 , a free space wavelength of $100 \mu \mathrm{m}$, and a perfectly conducting metal cladding. The waveguide width and thickness were chosen to be 150 and $10 \mu \mathrm{m}$, respectively. The simulation results are shown in Fig. 1(b); the facet reflectivity increases from $84 \%$ for the $\mathrm{TM}_{00}$ mode to $96 \%$ for the $\mathrm{TM}_{03}$ mode. The physical origin of the high facet reflectivity and subsequent low outcoupling efficiency for higher order lateral modes can be understood by considering the mode lobes as radiation sources at the laser facet: the lobes are separated by a distance much smaller than the free space wavelength due to the high mode confinement provided by MM waveguides [Fig. $1(c)]$. This results in strong destructive interference for emission into free space.

To calculate the threshold gain $g_{\text {th }}$ for the various waveguide modes, we used the expression $\Gamma g_{\mathrm{th}}=\alpha_{m}+\alpha_{\mathrm{wg}}$, where $\Gamma$ is the mode confinement factor, $\alpha_{\mathrm{wg}}$ is the waveguide loss, and $\alpha_{m}$ is the mirror loss calculated from the reflectivity data in Fig. 1(b). We first calculated $\alpha_{\mathrm{wg}}$ by solving for modes in the 150- $\mu \mathrm{m}$-wide waveguide structure depicted in Fig. 1(a) with and without $2.5-\mu \mathrm{m}$-wide side absorbers. We used the Drude model with relaxation constants $\tau=1 \mathrm{ps}$ and $\tau=0.1 \mathrm{ps}$ for lightly and heavily doped semiconductor layers, respectively. ${ }^{3}$ For the GaAs plasmon layers, $n$-doped at $5 \times 10^{18} \mathrm{~cm}^{-3}$, we calculate the refractive index to be $5.93+23.32 i$. The active region refractive index was calculated to be $3.49+0.0061 i$ and the gold refractive index was taken from Ref. 13 to be $240+400 i$. Waveguide losses from the 10 -nm-thick top titanium cladding layer were neglected. The simulations indicate that for MM waveguides without side absorbers, $\alpha_{\mathrm{wg}}$ and $\Gamma$ are approximately the same for all lateral mode orders due to the very strong confinement provided by the $\mathrm{MM}$ waveguide. For example, the $\mathrm{TM}_{00}$ and $\mathrm{TM}_{01}$ modes have $\alpha_{\mathrm{wg}}$ of 14.1 and $14.2 \mathrm{~cm}^{-1}$, respectively, and $\Gamma$ of $99 \%$ each. On the other hand, we obtained very different $\alpha_{\mathrm{wg}}$ for different lateral modes in the MM waveguide with side absorbers. The threshold gain calculated for different lateral modes in a 1.3 - mm-long MM waveguide with and without side absorbers is plotted in Fig. 1(d). For the waveguide without side absorbers, the $\mathrm{TM}_{00}, \mathrm{TM}_{01}$, and $\mathrm{TM}_{02}$ lateral modes all have thresholds of within $0.1 \mathrm{~cm}^{-1}$ of each other, indicating little mode discrimination between the $\mathrm{TM}_{00}$ and higher order lateral modes. For the waveguide with side absorbers, the $\mathrm{TM}_{00}$ mode clearly has the lowest threshold gain of all the lateral modes with mode discrimination on the order of a few $\mathrm{cm}^{-1}$. Furthermore, the side absorbers introduce very small additional loss to the $\mathrm{TM}_{00}$ mode.

To demonstrate the improvement of power output from devices with side absorbers, we processed and characterized MM waveguide terahertz QCLs with and without side absorbers. The QCL material was grown by molecular beam Downloaded 23 Jan 2008 to 128.103.60.225. Redistribution subject
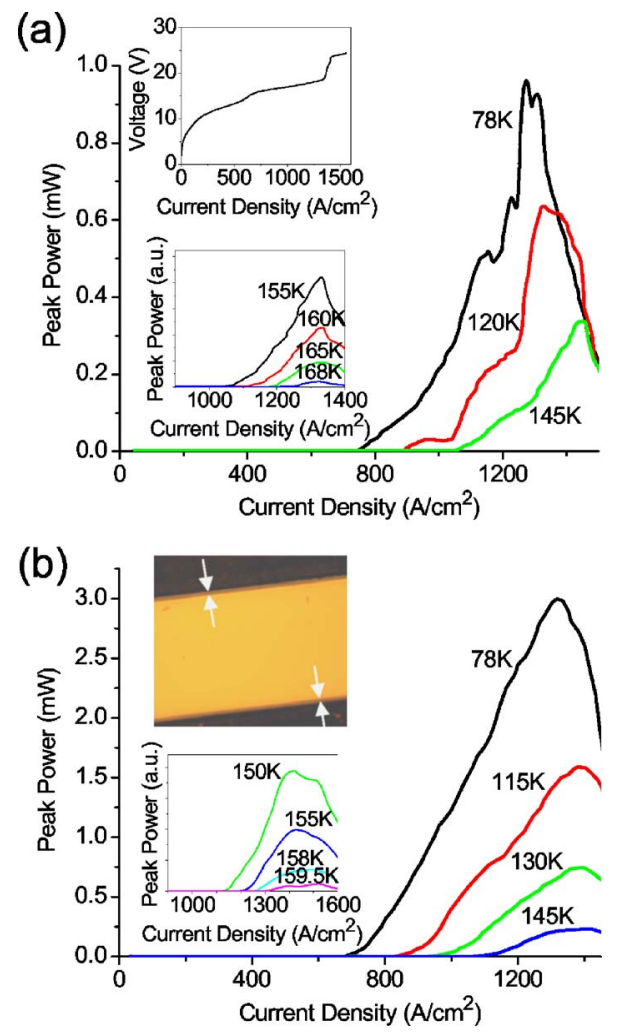

FIG. 2. (Color online) (a) Light output as a function of current ( $L I)$ of a representative MM waveguide device with no side absorbers. Upper inset: current-voltage characteristics of the device at $78 \mathrm{~K}$. Lower inset: $L I$ characteristics of the device close to the maximum operating temperature. (b) $L I$ characteristics of a representative MM waveguide device with side absorbers. Upper inset: image of the top of a MM waveguide device with side absorbers delineated by arrows. Lower inset: $L I$ characteristics of the device close to the maximum operating temperature.

epitaxy; the growth started with a 250 -nm-thick GaAs buffer layer, followed by a 300 -nm-thick $\mathrm{Al}_{0.5} \mathrm{Ga}_{0.5} \mathrm{As}$ layer, a 75 -nm-thick layer of GaAs $n$-doped to $5 \times 10^{18} \mathrm{~cm}^{-3}, 226 \frac{1}{2}$ stages of the active region design similar to that of Ref. 14 with a lower doping sheet density of $n_{s}=2.75 \times 10^{10} \mathrm{~cm}^{-2}$, and finally a 50-nm-thick GaAs layer $n$-doped to 5 $\times 10^{18} \mathrm{~cm}^{-3}$. The material was processed into gold $\mathrm{MM}$ waveguides following the procedure outlined in Refs. 4 and 5 . The $150-\mu \mathrm{m}$-wide ridges were defined via dry etching with a Microposit SU-8 2005 photoresist mask. After SU-8 removal, metal ( $\mathrm{Ti} / \mathrm{Au}, 10 / 200 \mathrm{~nm}$ ) was evaporated on top of the laser ridges. Side absorbers were fabricated on some devices by depositing photoresist onto the ridges, opening thin strips along the ridge edges with optical lithography, and removing the strips of gold and titanium by wet etching. The final side absorbers were measured to be approximately 3 - $\mu$ m-wide [inset of Fig. 2(b)]. The processed wafers were cleaved into approximately 1.3 -mm-long bars and indium mounted on gold-plated copper blocks.

The devices were placed in a helium flow cryostat and terahertz radiation was detected with a calibrated heliumcooled silicon bolometer. Light was collected with two 2 in. parabolic mirrors: one with a $5 \mathrm{~cm}$ focal length to collimate the light from the source, and another, $5 \mathrm{~cm}$ away from the first one, with a $15 \mathrm{~cm}$ focal length to refocus the light into the bolometer. The devices were operated in pulsed mode with $30 \mathrm{~ns}$ pulses at a $100 \mathrm{kHz}$ repetition rate, with an additional $600 \mathrm{~Hz}$ modulation for lock-in detection. The light tional $600 \mathrm{~Hz}$ modulation for lock-in detection. The light
to AIP license or copyright; see http://apl.aip.org/apl/copyright.jsp 


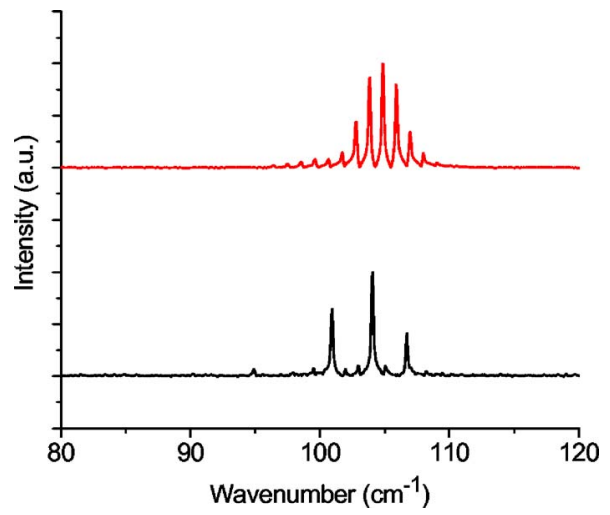

FIG. 3. (Color online) Representative spectra of lasers with (top) and without (bottom) side absorbers operating at a temperature of $78 \mathrm{~K}$ and a current density of $900 \mathrm{~A} / \mathrm{cm}^{2}$.

intensity-current $(L I)$ and current-voltage (IV) characteristics of a representative device without side absorbers are shown in Fig. 2(a) for different heatsink temperatures. At a heatsink temperature of $78 \mathrm{~K}$, the threshold current density was measured to be $710 \mathrm{~A} / \mathrm{cm}^{2}$ and the maximum peak power was measured to be $0.92 \mathrm{~mW}$. The $L I$ characteristics of a representative device with side absorbers are shown in Fig. 2(b). The threshold current density for this device at the heatsink temperature of $78 \mathrm{~K}$ was $685 \mathrm{~A} / \mathrm{cm}^{2}$, similar to that for devices without side absorbers, and the maximum peak power was measured to be $3.0 \mathrm{~mW}$, which is over three times larger compared to that from devices without side absorbers.

Representative emission spectra of the devices with and without side absorbers are shown in Fig. 3. The mode spacing in the spectra of the device without side absorbers is nonperiodic, indicating lasing in multiple lateral order modes. In comparison, devices with side absorbers showed emission spectra with mode spacing indicative of Fabry Perot lasing under a single lateral mode. The spectra and enhanced power outcoupling indicate that the side absorbers enforce lasing in the $\mathrm{TM}_{00}$ mode.

The devices without side absorbers operated up to a maximum temperature of $168 \mathrm{~K}$ [see Fig. 2(a)], which is the highest reported temperature for terahertz QCLs processed with In/ Au bonding. We note that the gold MM waveguide devices reported in the original publication ${ }^{14}$ operated only up to $142 \mathrm{~K}$. The improved performance of our devices may stem from better growth and/or processing quality, as well as lower doping density. The devices with side absorbers had a slightly worse temperature performance, with a maximum operating temperature of $\approx 160 \mathrm{~K}$ [see Fig. 2(b)]. This reduction in temperature performance is attributed to the small amount of losses introduced by the side absorbers. The exceptional temperature performance of our devices demonstrates the potential of a three-well design ${ }^{14}$ for increasing the maximum operating temperature of terahertz QCLs. We note that the temperature performance of our devices may be further boosted ${ }^{15}$ by using a copper cladding since copper has better thermal ${ }^{16}$ and optical ${ }^{13}$ properties than gold.

J.F., M.B., and F.C. acknowledge support from the AFOSR under Contract No. FA9550-05-1-0435 (Gernot Pomrenke). The structures were processed in the Center for Nanoscale Science (CNS) in Harvard University. HarvardCNS is a member of the National Nanotechnology Infrastructure Network. J.F. acknowledges support from the NSF Graduate Fellowship. S.K., M.L., G.D., and E.L. acknowledge support from EPSRC (UK) and Her Majesty's Government Communications Centre.

${ }^{1}$ B. S. Williams, Nat. Photonics 1, 517 (2007).

${ }^{2}$ R. Köhler, A. Tredicucci, F. Beltram, H. E. Beere, E. H. Linfield, A. G. Davies, D. A. Richie, R. C. Iotti, and F. Rossi, Nature (London) 417, 156 (2002).

${ }^{3}$ S. Kohen, B. S. Williams, and Q. Hu, J. Appl. Phys. 97, 053106 (2005).

${ }^{4}$ K. Unterrainer, R. Colombelli, C. Gmachl, F. Capasso, H. Y. Hwang, D. L. Sivco, and A. Y. Cho, Appl. Phys. Lett. 80, 3060 (2002).

${ }^{5}$ B. S. Williams, S. Kumar, H. Callebaut, Q. Hu, and J. Reno, Appl. Phys. Lett. 83, 2124 (2003).

${ }^{6}$ G. Scalari, N. Hoyler, M. Giovannini, and J. Faist, Appl. Phys. Lett. 86, 181101 (2005).

${ }^{7}$ A. W. M. Lee, Q. Qin, S. Kumar, B. Williams, and Q. Hu, Appl. Phys. Lett. 89, 141125 (2006).

${ }^{8}$ J. Darmo, V. Tamosiunas, G. Fasching, J. Kroll, K. Unterrainer, M. Beck, M. Giovannini, J. Faist, C. Kremser, and P. Debbage, Opt. Express 12, 1879 (2004).

${ }^{9}$ A. W. M. Lee, Q. Qin, S. Kumar, B. S. Williams, Q. Hu, and J. Reno, Opt. Lett. 32, 2840 (2007).

${ }^{10}$ J. A. Fan, M. A. Belkin, F. Capasso, S. Khanna, M. Lachab, A. G. Davies, and E. H. Linfield, Opt. Express 14, 11672 (2006).

${ }^{11}$ S. Kumar, B. S. Williams, Q. Qin, A. W. M. Lee, Q. Hu, and J. Reno, Opt. Express 15, 113 (2007).

${ }^{12}$ G. Scalari, L. Sirigu, R. Terazzi, C. Walther, M. I. Amanti, M. Giovannini, N. Hoyler, J. Faist, M. L. Sadowski, H. Beere, D. Ritchie, L. A. Dunbar, and R. Houdre, J. Appl. Phys. 101, 081726 (2007).

${ }^{13}$ M. A. Ordal, L. L. Long, R. J. Bell, S. E. Bell, R. R. Bell, R. W. Alexander, Jr., and C. A. Ward, Appl. Opt. 22, 1099 (1983).

${ }^{14}$ H. Luo, S. R. Laframboise, Z. R. Wasilewski, G. C. Aers, H. C. Liu, and J. C. Cao, Appl. Phys. Lett. 90, 041112 (2007).

${ }^{15}$ M. A. Belkin, J. A. Fan, F. Capasso, S. Khanna, M. Lachab, A. G. Davies, and E. H. Linfield, Opt. Express (to be published).

${ }^{16}$ B. S. Williams, S. Kumar, Q. Hu, and J. Reno, Opt. Express 13, 3331 (2005). 